H I G H L I G H T S

GENE EXPRESSION

\section{The complete picture}

Gene-expression events are intricately linked - and there is now a way to view the complete set of events together in a single living cell. Reporting in Cell, David Spector and colleagues have developed a system to simultaneously visualize gene expression at the DNA, RNA and protein levels.

Spector and co-workers constructed an inducible plasmid with a transcriptional unit that encodes cyan fluorescent protein $(\mathrm{CFP})$ - allowing visualization of the protein - and socalled translational operator RNA (a 19-nt RNA stem loop). Upstream of the transcriptional unit are repeats of the bacterial lac operator sequence, so that, in the presence of a lac-repressorbinding protein that is expressed as a fluorescent fusion protein, the transgene locus can be visualized. And, transcribed RNA can be visualized by expression of yellow fluorescent protein (YFP) fused to MS2, a protein that binds the translational operator RNA. The authors selected a stable transfectant that expressed $\sim 200$ copies of the transgene that was integrated in a euchromatic region in human chromosome 1.

The authors simultaneously analysed the chromatin and RNA dynamics at the transcription site, and showed that, in the absence of transcriptional activation, the CFP-lac repressor marks the chromatin of the transgene locus as a highly condensed dot and MS2-YFP is diffusely distributed throughout the nucleus. On transcriptional induction, the locus decondenses, proteins of the geneexpression machinery colocalize with the decondensed locus, and MS2-YFP associates with the nascent transcripts at the locus and with messenger ribonucleoprotein (mRNP) particles in the nucleoplasm.

Next, Spector and colleagues measured the kinetics of RNA synthesis at the site of transcription. They detected an early increase in RNA levels (5 min post-induction), as shown by a small spot of MS2-YFP at the locus, while the chromatin was still highly con- densed. The first chromatin changes were detected at $\sim 17.5 \mathrm{~min}$, by which time transcripts were seen across the entire locus. RNA levels peaked by $\sim 130$ min and subsequently decreased: the induction of transcription seems to slow over time.

Heterochromatin protein 1 (HP1), as well as histone $\mathrm{H} 3$ that is trimethylated at lysine 9 (this modification serves as the HP1-binding site), colocalize with the condensed locus, which indicates that it is heterochromatic in nature. After transcriptional activation, HP1 and tri-methylated histone $\mathrm{H} 3$ are depleted while the locus becomes decondensed, and the histone variant $\mathrm{H} 3.3$ becomes deposited. So, histone exchange might be a possible mechanism for removing methylated histones from chromatin during transcriptional activation.

Spector and colleagues have developed a powerful tool for studying gene expression and chromatin, in time and space, in living cells. They suggest that this system of transgene arrays might also prove useful for studying the mechanisms of RNA-interferencemediated gene silencing in vivo. Arianne Heinrichs, Editor,

Nature Reviews Molecular Cell Biology

\section{(4) References and links} ORIGINAL RESEARCH PAPER Janicki, S. M. et al. From silencing to gene expression: real-time analysis in single cells. Cell 116, 683-698 (2004) WEB SITE

David Spector's laboratory: http://spectorlab.cshl.edu

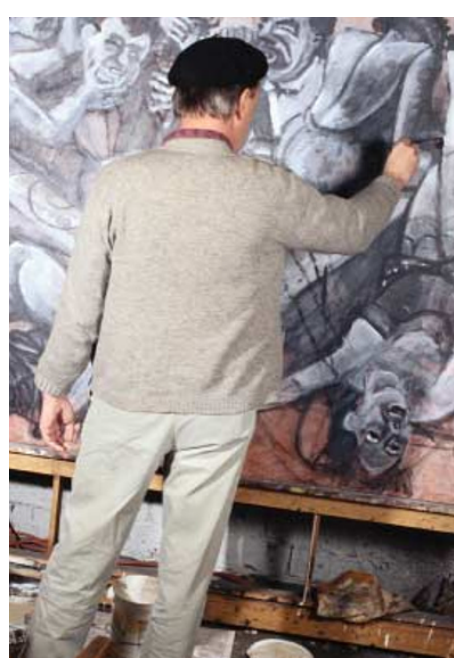

\section{IN BRIEF}

\section{GENE THERAPY}

Reengineered salivary glands are stable endogenous bioreactors for systemic gene therapeutics.

Voutetakis, A. et al. Proc. Natl Acad. Sci. USA 101, 3053-3058 (2004)

The authors show that salivary glands have potential as a target tissue for gene therapy, and could circumvent the safety concerns that surround the targeting of critical-for-life organs. They administered low doses of adeno-associated virus vector encoding human erythropoietin (hEPO) to mouse salivary glands. Encouragingly, vector DNA was only found in the target salivary glands, and the increased serum hEPO levels were stable and vector dependent.

\section{TECHNOLOGY}

Guidelines for the selection of highly effective siRNA sequences for mammalian and chick RNA interference.

Ui-Tei K. et al. Nucleic Acids Res. 32, 936-948 (2004)

Most siRNAs simply do not do what researchers want them to do: that is, to silence genes in an effective sequence-specific fashion. Using various gene targets and cells, Kumiko Ui-Tei and colleagues systematically analysed the gene-silencing abilities of siRNAs and deduced four rules for selecting those that work in mammal and chick cells. It seems that the nucleotides that lie towards the end of the sense and antisense strands are most important to the success of siRNAs.

\section{STATISTICAL GENETICS}

A general framework for analysing the genetic architecture of developmental characteristics.

Wu, R. et al. Genetics (in the press)

The growth of an organism is shaped by the way genes interact with each other and with the environment. The authors of this paper have developed and tested a statistical model that maps the quantitative trait loci that affect the trajectory of development and have quantified their effect; importantly, the model also measures the contribution of epistasis to growth at various stages of development.

\section{EVOLUTION}

High $\mathrm{MHC}$ diversity maintained by balancing selection in an otherwise genetically monomorphic mammal.

Aguilar, A. et al. Proc. Natl Acad. Sci. USA 27 Feb 2004 (doi:10.1073/pnas.0306582101)

Although the San Nicolas island fox is the most genetically monomorphic among sexually reproducing mammals, it has high levels of variation at MHC loci. Using a simulation, the authors show that this could arise following a severe bottleneck in the population's past, combined with unusual selection coefficients. These results have implications for conservation genetics: inbreeding in small populations of endangered species might best be minimized by preserving diverse fitness-related genes as well as neutral variation. 